\title{
COMPUTATIONAL STUDY ON BREAK-UP MECHANISMS OF ISOLATED VAPOUR SLUGS DURING SATURATED FLOW BOILING CONDITIONS
}

\author{
Manolia Andredaki ${ }^{1}$, Anastasios Georgoulas ${ }^{1 *}$, Daniele Mangini ${ }^{1}$, Lucio Araneo ${ }^{2}$, Luca Pietrasanta ${ }^{1}$, \\ Nicolas Miché ${ }^{1}$, Marco Marengo ${ }^{1}$ \\ ${ }^{1}$ Advanced Engineering Centre, School of Computing Engineering and Mathematics, Cockcroft Building, \\ Lewes Road, University of Brighton, Brighton BN2 4GJ, UK \\ ${ }^{2}$ Politecnico di Milano, Dipartimento di Energia, Via Lambruschini 4A, 20158 Milano, Italy
}

\begin{abstract}
In the present paper, an enhanced Volume Of Fluid (VOF) based numerical simulation framework is applied for the conduction of parametric numerical experiments, aiming to investigate break-up phenomena of elongated vapour slugs, within circular mini-channels. The effect of fundamental controlling parameters, in the resulting break-up characteristics, is examined. In more detail, four different series of parametric numerical experiments of isolated liquid slugs within a mini-channel were performed, investigating the effects of Applied Pressure Drop (APD), Surface Tension Coefficient (STC), Initial Liquid Film Thickness (ILFT) and Applied Heat Flux (AHF), on the vapour slug dynamics. Such break-up phenomena have been observed experimentally, in the past, within mini-channel branches of a hybrid thermosyphon/pulsating heat pipe device, under micro-gravity conditions. The simulation results identify three prevailing regimes. A "full break-up" regime, a "partial break-up" regime and a "no break-up" regime. It is characteristic that an entrainment of liquid droplets at the trailing edge of the vapour slugs, is responsible for their subsequent "full break-up", into a leading and a trailing bubble in each case, as it is identified from the numerical simulations. Moreover, it is quite interesting that the value of the AHF, does not seem to influence the resulting break-up regime and its main characteristics.
\end{abstract}

KEY WORDS: Two-phase/Multiphase flow, Boiling and evaporation, Heat pipe, Computational methods.

\section{INTRODUCTION}

The demand for increasingly higher performances of electronic equipment, has pushed researchers and engineers to develop a new generation of heat dissipation systems, based on the local phase-change of a working fluid. Efficient thermal control, especially in space applications, and the need to reduce mechanical elements have become of crucial importance. Two-phase, closed-loop, wickless heat pipes, such as a ThermoSyphon (TS) or a Pulsating Heat Pipe (PHPs), that constitute the motivation of the present numerical investigation, can meet such requirements. In the work of Mangini et al. [1], a novel concept of a hybrid TS/PHP with a diameter bigger than the capillary limit is tested, both on ground and in hyper/micro gravity conditions, during the 61st ESA Parabolic Flight Campaign. The device was filled with FC-72 and it was made of an aluminium tube (I.D. $3 \mathrm{~mm}$ ) bent into a planar serpentine. The proposed setup and some indicative flow visualization results during the normal gravity, hyper-gravity and microgravity stages of a single parabola, are depicted in Figure 1. As it can be observed during the $1 \mathrm{~g}$ and $1.8 \mathrm{~g}$ periods the developed two-phase flow within the visualisation channel of the proposed device is stratified, while during the microgravity period $(0 \mathrm{~g})$ the flow is transformed into a slug/plug flow. However, during the micro-gravity period, the high-speed images from the experiments, reveal the development of intense capillary waves and other types of perturbations in the liquid film between the elongated vapour bubbles and the channel wall, that in most cases are accompanied by the break-up of the vapour slugs, in the vicinity of their trailing edges, either into two subsequent elongated vapour slugs or into a main elongated vapour slug with small bubbles detaching from its trailing edge. The present investigation aims to give further insight on these observed phenomena, by identifying and quantifying

*Corresponding Author: a.georgoulas@brighton.ac.uk 
break-up regimes in the case of isolated elongated vapour slugs within circular mini-channels, in adiabatic and saturated flow boiling conditions, within a mini-channel with the same characteristics as the mini-channel branches of the proposed hybrid TS/PHP device. For this purpose, an Enhanced VOF method that has been developed in the context of OpenFOAM CFD Toolbox, coupled with heat transfer and phase-change, is applied in order to perform a series of parametric numerical experiments, aiming to understand the various break-up mechanisms and characteristics. The proposed numerical framework has been presented in detail, extensively validated and successfully applied in the past for the case of quasi-static bubble growth and detachment from submerged orifices in isothermal liquid pools [2], as well as for cases of saturated pool boiling [3].
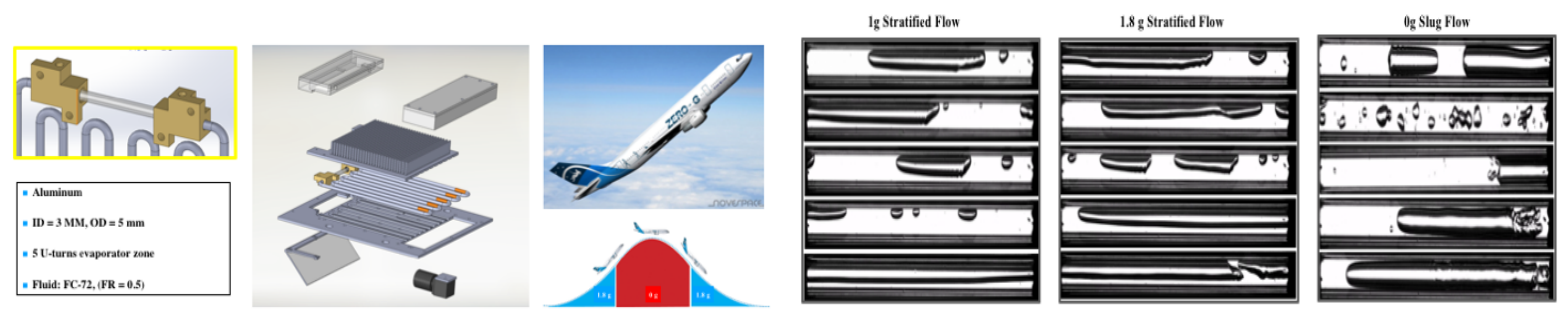

Fig. 1 Experimental two-phase flow visualization within a hybrid TS/PHP [1].

\section{INVESTIGATION METHODOLOGY}

\subsection{Numerical Method}

\section{Governing Equations}

In this section, the governing equations for mass, momentum, energy, and volume fraction are presented. It should be mentioned that liquid and vapour phases are both treated as incompressible, Newtonian fluids. The mass conservation equation is given as:

$$
\nabla \cdot(\rho \vec{U})=\dot{\rho}
$$

where $U$ is the fluid velocity and $\rho$ is the bulk density. The source term on the right-hand side accounts for the phase change. The conservation of momentum is given by the following equation:

$$
\frac{\partial}{\partial \mathrm{t}}(\rho \overrightarrow{\mathrm{U}})+\nabla \cdot(\rho \overrightarrow{\mathrm{UU}})-\nabla \cdot\left\{\mu\left[\nabla \overrightarrow{\mathrm{U}}+(\nabla \overrightarrow{\mathrm{U}})^{\mathrm{T}}\right]\right\}=-\nabla \mathrm{p}+\overrightarrow{\mathrm{f}}_{\mathrm{ST}}+\overrightarrow{\mathrm{f}}_{\mathrm{g}}
$$

where $\mathrm{p}$ is the pressure and $\mu$ is the bulk dynamic viscosity. The momentum source terms in the right-hand side of the equation account for the effects of surface tension and gravity, respectively. The surface tension term is modelled according to the classical approach of Brackbill et al. [4]. The conservation of energy balance is given by the following equation:

$$
\frac{\partial}{\partial \mathrm{t}}\left(\rho \mathrm{c}_{\mathrm{p}} \mathrm{T}\right)+\nabla \cdot\left(\overrightarrow{\mathrm{U}} \rho \mathrm{c}_{\mathrm{p}} \mathrm{T}\right)-\nabla \cdot(\lambda \nabla \mathrm{T})=\dot{\mathrm{h}}
$$

where $c_{p}$ is the bulk heat capacity, $T$ the temperature field, and $\lambda$ is the bulk thermal conductivity. The source term on the right-hand side of the equation represents the contribution of the enthalpy of evaporation/condensation or else the cooling/heating associated with the latent heat of the phase-change. The volume fraction $\alpha$ is advected by the flow field by the following equation:

$$
\frac{\partial \alpha}{\partial \mathrm{t}}+\nabla \cdot(\alpha \overrightarrow{\mathrm{U}})-\nabla \cdot\left(\alpha(1-\alpha) \mathrm{U}_{\mathrm{r}}\right)=\frac{\dot{\rho}}{\rho} \alpha
$$

Interface sharpening is very important in simulating two-phase flows of two immiscible fluids. In OpenFOAM the sharpening of the interface is achieved artificially by introducing the extra compression term $(\nabla$. $\left.\left(\alpha(1-\alpha) U_{r}\right)\right)$ in Equation (4). $U_{r}$ is an artificial compression velocity. The divergence of the compression velocity $U_{r}$, ensures the conservation of the volume fraction $\alpha$, while the term $\alpha(1-\alpha)$ limits this artificial 
compression approach only in the vicinity of the interface, where $0<\alpha<1$ [5]. The level of compression depends on the value of $\mathrm{C}_{\gamma}([5]$, [6]). For the simulations of the present investigation, initial, trial simulations indicated that a value of $\mathrm{C}_{\gamma}=1$ should be used, in order to maintain a quite sharp interface without at the same time having unphysical results. The source term on the right-hand side of the Equation (4) is needed because, due to the local mass source terms, the velocity field is not free of divergence. Finally, the bulk fluid properties $\gamma$ are computed as the averages over the liquid $\left(\gamma_{1}\right)$ and vapour $\left(\gamma_{v}\right)$ phases, weighted with the volume fraction $\alpha$. The VOF-based solver that is used in the present investigation has been modified accordingly in order to account for an adequate level of spurious currents suppression. More details on the proposed validation as well as on the proposed improved VOF method can be found in the paper by Georgoulas et al [2].

\section{Phase Change Model}

The utilized phase change model that was implemented in the improved OpenFOAM VOF solver that is used in the present investigation, will be described briefly in this section. Supplementary details can be found in the work by Georgoulas et al. [3]. In the case of evaporation, the evaporating mass flux at the liquid-vapour interface $j_{\text {evap }}$ is calculated from the following equation:

$$
\mathrm{j}_{\text {evap }}=\frac{\mathrm{T}_{\mathrm{int}}-\mathrm{T}_{\mathrm{sat}}}{\mathrm{R}_{\mathrm{int}} \mathrm{h}_{\mathrm{lv}}}
$$

where $T_{\text {int }}$ is the temperature of the interface, $T_{\text {sat }}$ is the saturation temperature, $R_{\text {int }}$ is the interfacial heat resistance and $h_{\mathrm{lv}}$ is the latent heat of evaporation at the saturation temperature. The amount of liquid that evaporates is calculated locally and the resulting source term field is smeared over a few cells in order to avoid numerical instabilities. The evaporating mass is taken away on the liquid side of the interface and reappears on the vapour side, following the process suggested in the work of Hardt and Wondra [7]. Further details on the proposed process can be found in the work of Georgoulas et al. [3].

\subsection{Validation of Numerical Method}

As mentioned above, the utilised enhanced VOF-based numerical model, has been extensively validated in the past against existing analytical solutions and experimental data on adiabatic and diabatic bubble and droplet dynamics [2], [3], [8]. For the purposes of the present investigation, it was deemed appropriate to perform an additional validation against the experimental data on Taylor bubble development in a mini-channel T-junction that are reported in the work of Arias et al. [9]. This was decided in order to ensure that the proposed VOFbased method can accurately predict different regimes of two phase flow development, since previous validation of the method was mainly with experiments of quasi-static bubble growth in stagnant liquid domains and pool boiling. The 3D computational domain, the computational mesh and the applied boundary conditions for the proposed validation cases are illustrated in Figure 2.

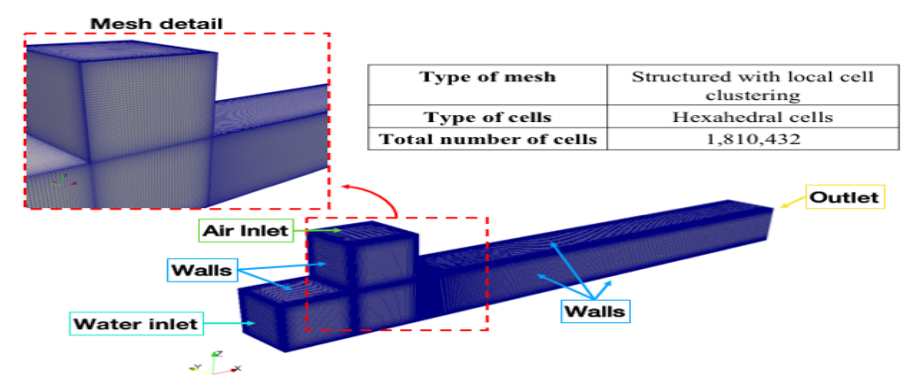

Fig. 2 Computational domain, mesh and boundary conditions, utilised in the validation runs.

The initial conditions and the main characteristics of the experimental runs that were reproduced numerically for the proposed additional validation of the model, are summarised in Table 1 . The working fluids properties are taken as water and air at ambient conditions, for the liquid and the gas phases, respectively and fully wetted conditions are applied in the channel walls. 
Table 1 Initial conditions of validation runs, reported in the work of Arias et al. [9].

\begin{tabular}{|c|c|c|}
\hline & $\mathbf{U}_{\text {water }}[\mathbf{m} / \mathbf{s}]$ & $\mathbf{U}_{\text {air }}[\mathbf{m} / \mathbf{s}]$ \\
\hline Case 1 & 0.106 & 0.344 \\
\hline Case 2 & 0.531 & 0.068 \\
\hline Case 3 & 0.318 & 0.242 \\
\hline
\end{tabular}

A comparison of temporal and spatial evolution of the generated bubbles in each case, between the numerical model predictions (present investigation) and the experimental high-speed visualization images from the work of Arial et al. [9], is shown in Figure 3.
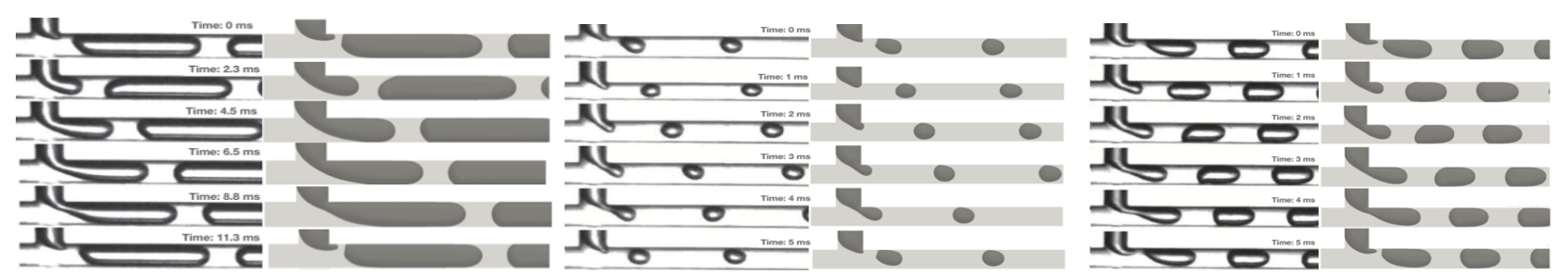

Fig. 3 Comparison of experimental (left) [9] and numerical (right) results (present investigation), for the three different cases of Taylor bubble generation in the considered mini-channel T-junction.

As it can be observed the numerical model accurately predicts the development of the different two-phase flow regimes in relation to the applied gas/liquid flow rates at the inlets of the T-junction.

\section{APPLICATION OF THE VALIDATED NUMERIVCAL METHOD FOR THE SIMULATION OF BREAK-UP CHARACTERISTICS}

In the current section of the present work, the validated numerical model is further applied for the conduction of a wide number of parametric numerical simulations, aiming to identify and quantify the effects of fundamental controlling parameters, in the resulting break-up regimes for the case of isolated elongated vapour slugs, within adiabatic and heated mini-channels. In more detail, two different working fluids (FC-72 and Ethanol) and two different channel diameters $(3 \mathrm{~mm}$ and $2 \mathrm{~mm})$ are considered, with a variation in the APD between the inlet and the outlet of the consider mini-channels, a "virtual" variation of the STC and a variation of the ILFT between the vapour slugs and the channel wall. All these cases are considered to be adiabatic with the fluid properties for the liquid and vapour phases taken at a saturation equilibrium point. However, 12 additional simulations are performed by applying two different heat flux values in the central part of the considered circular mini-channel, in order to see the resulting break-up regimes for the case of adiabatic flows as well. The overall parametric analysis consists of 88 simulations. Initially, in all cases, a prescribed pressure drop is assigned between the inlet and the outlet and a single phase simulation is performed until a steady state flow is established within the channel. Then a vapour slug is patched close to the inlet of the channel, that is then carried away downstream by the previously developed liquid flow. The initial vapour slug length in all cases is taken as three times the mini-channel diameter. The initial film thickness for the base case is taken as $50 \mu \mathrm{m}$. In all cases the bubbles are initiated $1.55 \mathrm{~mm}$ from the inlet of the channel, as a combination of a central cylinder with two superimposed spheres at the edges.

\subsection{Computational Set-up}

As a first step, and since the focus of the investigation is typical elongated vapour slugs from a slug flow regime, an axisymmetric computational domain was constructed for the proposed parametric analysis. The adopted computational domain, mesh and boundary conditions are illustrated in Fig. 4. As it can be seen, a wedge type geometry was constructed representing a $5^{\circ}$ section of the corresponding $3 \mathrm{D}$ domain in the considered physical problem. In total two different circular mini-channels are considered having a total length of $90 \mathrm{~mm}$ and a diameter of $3 \mathrm{~mm}$ and $2 \mathrm{~mm}$ respectively. A non-uniform structured computational mesh with local refinement close to the walls of the channel was used in each case, consisting of 840,000 and 666,000 hexahedral cells, respectively. A minimum cell size of $1 \mu \mathrm{m}$ (close to the channel walls) and a maximum cell 
size of $15 \mu \mathrm{m}$ (in the vicinity of the axis of symmetry) were selected after the conduction of a mesh independency study. The overall domain sizes in the XY plane in each case are $90 \mathrm{~mm} \times 3 \mathrm{~mm}$ and $90 \mathrm{~mm} \times$ $2 \mathrm{~mm}$, for the channel with the $3 \mathrm{~mm}$ and $2 \mathrm{~mm}$ diameter, respectively. The $3 \mathrm{~mm}$ diameter channel was selected since the base case for the proposed parametric analysis, aims to imitate, as much as possible, the main conditions of the hybrid TS/PHP microgravity experiments reported in the work of Mangini et al. [1]. The length of $90 \mathrm{~mm}$ was selected in order to safely allow the initiated vapour slugs to travel downstream from their initiation point for a certain distance, within a previously developed single phase flow, in order to capture the resulted liquid film dynamics and the potential break-up regimes. The second channel with the $2 \mathrm{~mm}$ diameter as well as the second working fluid (Ethanol) were selected just for enhancing the present parametric analysis.

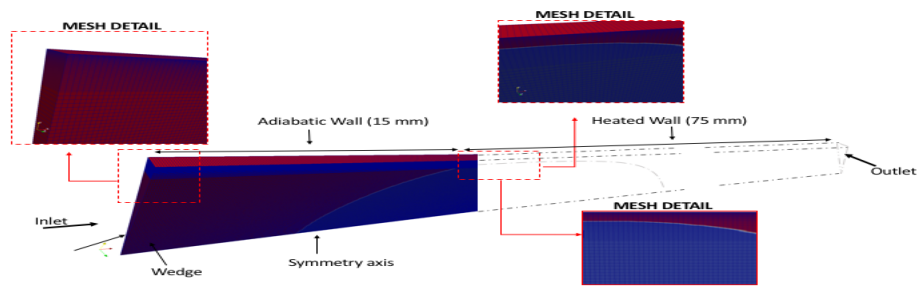

Fig. 4. Computational domain, mesh and boundary conditions.

\subsection{Mesh Independency Study}

In order to adopt the proposed mesh characteristics, a mesh-independency study was carried out for four different mesh densities, for the case of $3 \mathrm{~mm}$ diameter mini-channel. The adopted mesh for the present numerical investigation, consists of 840,000 cells. $2 \mathrm{D}$ axisymmetric simulations for the case of $60 \mathrm{~Pa}$ pressure drop with a coarser mesh of 420,000 cells, a finer mesh of 1,260,000 cells and an even finer mesh of 1,680,000 cells were also performed. The results are shown in Fig.5.

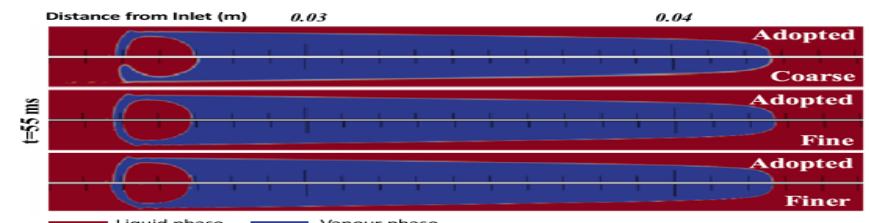

Fig. 5. Comparison between the numerical model predictions using the adopted mesh vs the coarse mesh (top), the adopted mesh vs the fine mesh (middle) and the original mesh vs the finest mesh (bottom) for the time $55 \mathrm{~ms}$.

As it can be observed, the adopted mesh characteristics ensure a mesh independent solution and therefore can be safely used for the purposes of the present parametric analysis.

\subsection{Parametric Numerical Simulations}

In the present section of the current paper, the effect of each of the considered controlling parameters is illustrated indicatively, comparing the resulting break-up regimes for some of the overall runs of the present parametric numerical experiments. In the end, all of the 88 simulated cases are gathered according to the resulting break-up regime, composing a dimensionless flow map.

\section{Effect of Applied Pressure Drop}

In Figure 6, the effect of pressure drop for three cases with a different APD at the ends of the $3 \mathrm{~mm}$ diameter mini-channel is depicted, indicatively. In all three cases the working fluid (FC-72), the ILFT, the STC and the AHF are kept constant. It is obvious that even small variations in the APD can lead to significant outputs in the resulting bubble break-up regime. In the first case $(\Delta \mathrm{P}=15 \mathrm{~Pa})$ quite intense capillary waves are evident within the liquid film that surrounds the elongated vapour slug close to its trailing edge up to the end of the simulation without any kind of bubble break-up being identified. At this point it should be mentioned that for 
all cases considered the duration of the simulation is $90 \mathrm{~ms}$. This behaviour from now on it will be referred as "no break-up" regime and it constitutes the first of the three in total observed regimes among the overall considered cases. With the further increase of the APD $(\Delta \mathrm{P}=45 \mathrm{~Pa})$, apart from the aforementioned capillary waves, the interface at the trailing edge of the bubble fluctuates significantly with the maximum fluctuation located at the centre axis of the channel that at time $\mathrm{t}=59.6 \mathrm{~ms}$ leads to the formation of small spherical bubbles that disintegrate successively from the fluctuating interface core. This type of partial bubble break-up constitutes the second of the three observed regimes. From now on this will be referred as "partial break-up" regime. Finally, the further increase of the APD $(\Delta \mathrm{P}=70 \mathrm{~Pa})$ a liquid jet starts to progressively penetrate the bubble from its trailing edge. This leads, at the end, to the encapsulation of a liquid drop within the elongated vapour bubble. The wobbling of the proposed liquid drop in the vertical direction, at $t=60.5 \mathrm{~ms}$, breaks the liquid film leading to a complete break-up of the bubble into an elongated leading part and a shorter trailing part. This break-up regime constitutes the third of the observed three regimes and it will be referred from now on as, "full break-up" regime.

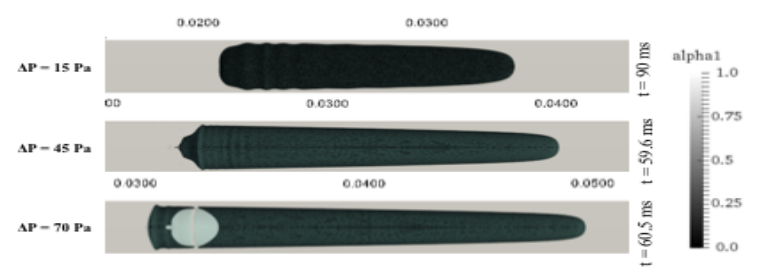

Fig. 6. The effect of the APD on the resulting elongated vapour slug break-up regime $\left(\mathrm{FC}-72, \delta_{\text {film }}=50 \mu \mathrm{m}\right.$, $\left.\mathrm{D}_{\text {channel }}=3 \mathrm{~mm}, \sigma=0.084 \mathrm{~N} / \mathrm{m}, \mathrm{q} "=0 \mathrm{~W} / \mathrm{m}^{2}\right)$.

\section{Effect of Surface Tension Coefficient}

In Figure 7, the effect of surface tension for three cases with a different STC for the $3 \mathrm{~mm}$ diameter minichannel is illustrated. In all three cases the working fluid (FC-72), the ILFT, the APD and the AHF are kept constant while the STC of the base case is "virtually" doubled and tripled keeping all the rest of the two-phase flow properties also constant. It can be noticed that as the STC increases the initially observed "full break-up" regime, successively shifts to "partial break-up" and "no break-up", respectively.

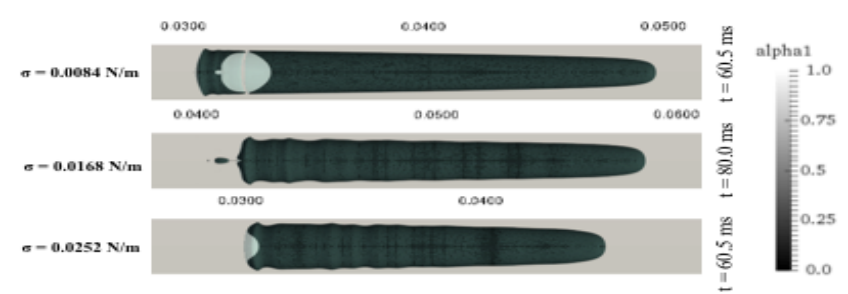

Fig. 7. The effect of the STC on the resulting elongated vapour slug break-up regime (FC-72, $\delta_{\text {film }}=50 \mu \mathrm{m}$, $\mathrm{D}_{\text {channel }}=3 \mathrm{~mm}, \Delta \mathrm{P}=70 \mathrm{~Pa}, \mathrm{q}$ ' $=0 \mathrm{~W} / \mathrm{m}^{2}$ ).

\section{Effect of Initial Liquid Film Thickness}

In Figure 8, the effect of film thickness for three cases with a different ILFT between the bubble and the wall of the $3 \mathrm{~mm}$ diameter mini-channel is depicted, indicatively. In all of the illustrated cases the working fluid (FC-72), the APD, the STC and the AHF are kept constant. As it can be observed, for relatively small values of the ILFT $(12-50 \mu \mathrm{m})$, the predicted break-up characteristics do not show any variation, since at exactly the same time instance, after the initiation of the two-phase flow $(\mathrm{t}=62 \mathrm{~ms})$, a similar liquid jet penetration that leads to a "full break-up" regime is observed. However, for further variations the effect becomes more significant $(\delta=75 \mu \mathrm{m}$ and $\delta=270)$.
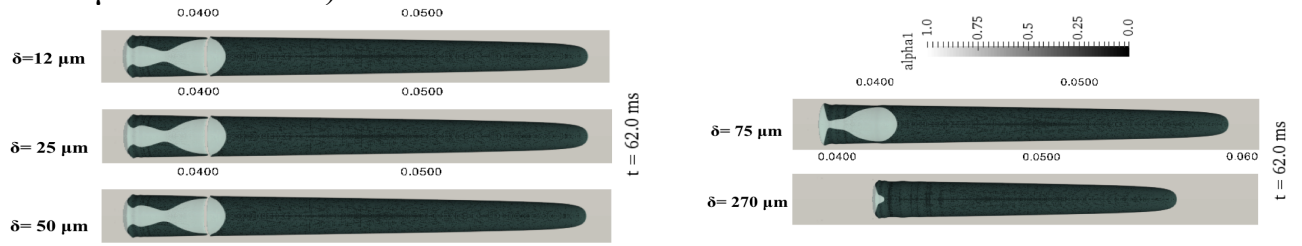

Fig. 8. The effect of the initial film thickness on the resulting elongated vapour slug break-up regime (FC-72, $\mathrm{D}_{\text {channel }}=3 \mathrm{~mm}, \Delta \mathrm{P}=60 \mathrm{~Pa}, \sigma=0.084 \mathrm{~N} / \mathrm{m}, \mathrm{q} "=0 \mathrm{~W} / \mathrm{m}^{2}$ ). 


\section{Effect of Applied Heat Flux}

In order to see if the previously hydro-dynamically generated regimes are altered, in the case of liquid film evaporation it was deemed appropriate to consider also some diabetic cases. For this purpose, six of the adiabatic cases were repeated with two different values of heat flux $\left(q "=5,000 \mathrm{~W} / \mathrm{m}^{2}\right.$ and $\left.\mathrm{q} "=10,000 \mathrm{~W} / \mathrm{m}^{2}\right)$. In Figure 9, the proposed effect is depicted for one of these 6 cases, indicatively. The following snapshots correspond to numerical runs with different $\mathrm{AHF}$ at the heated part of the $3 \mathrm{~mm}$ diameter mini-channel. Also in each of the illustrated cases the working fluid (FC-72), the APD, the STC and the ILFT are kept constant.

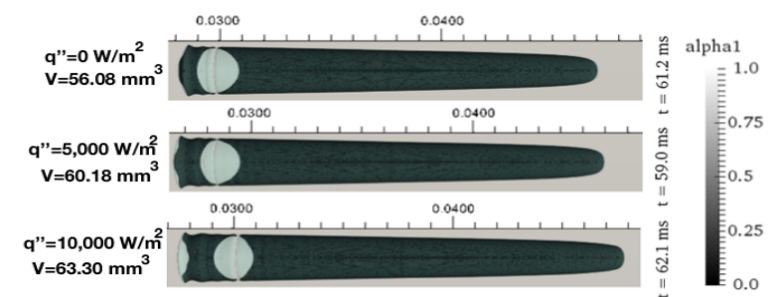

Fig. 9. The effect of the AHF in the heated section of the considered mini-channel on the resulting elongated vapour slug break-up regime (FC-72, $\delta_{\text {film }}=50 \mu \mathrm{m}, \mathrm{D}_{\text {channel }}=3 \mathrm{~mm}, \Delta \mathrm{P}=60 \mathrm{~Pa}, \sigma=0.084 \mathrm{~N} / \mathrm{m}$ ).

As it can be observed, it is quite interesting that despite the significant increase in the AHF and the corresponding resulting volume of the vapour due to the progressively higher liquid film evaporation, the bubbles in all three cases experience the same break-up regime and at similar time instances. This indicates that the identified break-up regimes are indeed related to the generated hydro-dynamic instabilities of the liquid film and the other observed inertial phenomena.

\section{Dimensional Analysis}

In order to quantify the global flow conditions that generate the previously identified main break-up regimes, a dimensional analysis is conducted. As it can be observed in Figure 10, the identified breakup regimes are grouped together forming a clear flow map using the Weber number (We) and a modified Froude number $\left(\mathrm{Fr}^{*}\right)$ in order to incorporate the effect of the applied pressure drop. In more detail, a central region where the bubbles undergo a "partial break-up" regime is identified with a bottom region of "no break-up" and a top region of "full break-up". The red and green circles indicate the previously mentioned six cases that were reproduced with an applied heat flux of 5,000 and 10,000 W/m², respectively. As it can be seen, all these heated cases end up with exactly the same regime as the corresponding adiabatic runs. This further strengthens the previous statement that the identified regimes seem to depend purely on the hydrodynamics of the two-phase flow and particular in the generated liquid film instabilities and the other identified inertia related phenomena at the trailing edge of the bubbles.

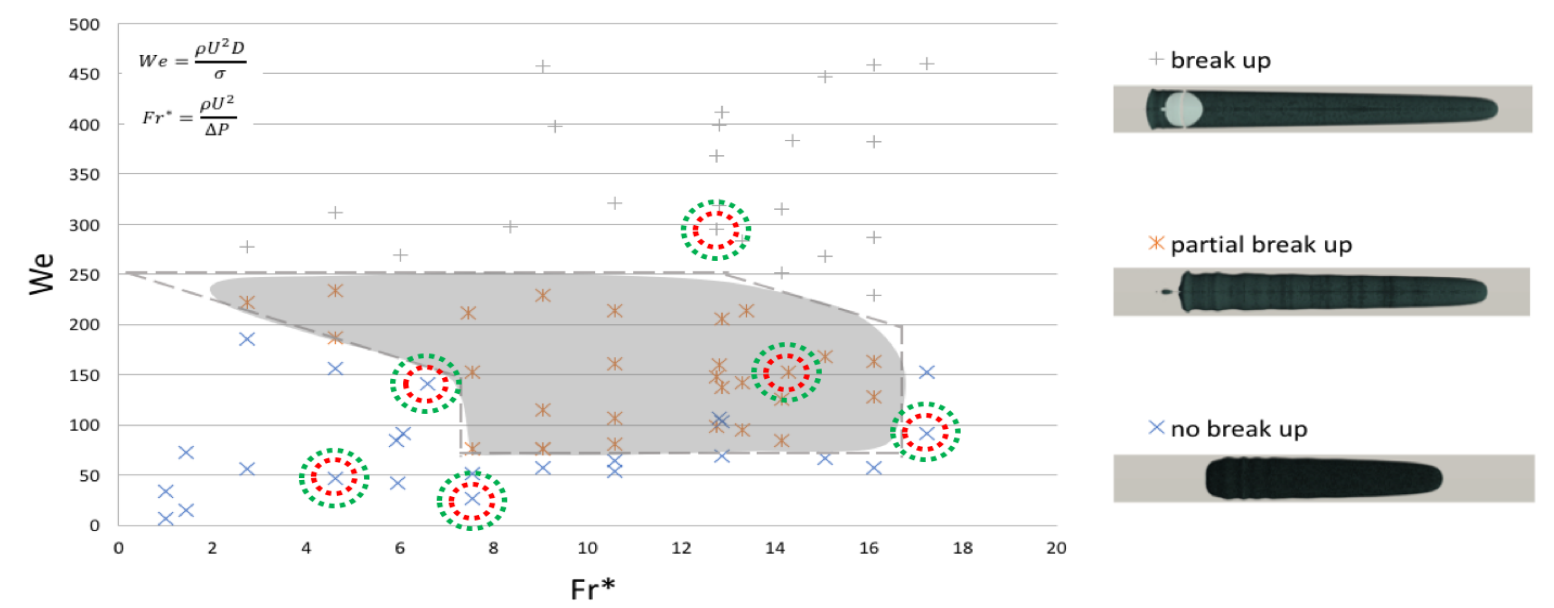

Figure 10. Flow map with different vapour slug break-up regimes (Weber number versus a modified Froude number). 


\section{CONCLUSIONS AND RECOMENDATIONS}

In the present work, an enhanced VOF-based numerical simulation framework is applied for the conduction of parametric numerical experiments, aiming to investigate the effect of various fundamental controlling parameters in the generated break-up phenomena in elongated vapour slugs, within circular mini-channels. The overall simulation results identify three prevailing regimes. A "full break-up" regime, a "partial break-up" regime and a "no break-up" regime. Some interesting phenomena are also revealed, such as the entrainment of liquid droplets at the trailing edge of the vapour slugs, that lead to the subsequent "full break-up" regime, as well as the presence of capillary waves at the liquid film surrounding the bubbles and other intense interface instabilities on their trailing edge. Another very interesting finding is the fact that the value of the AHF, does not seem to influence the resulting breakup regime and its main characteristics. Finally, a dimensional analysis led to the grouping of the identified break-up regimes according to the We and a modified Fr number of the initially imposed developed liquid flow. Such flow maps can constitute useful sub-models that can be incorporated in 1D simulation tools, in order to enhance their performance by implicitly accounting for the liquid film dynamics as well as for bubble break-up phenomena.

\section{ACKNOWLEDGMENT}

The Authors would like to acknowledge UK's Engineering and Physical Science Research Council support through the grant EP/P013112/1 as well as the ESA MAP Project INWIP. The authors would also like to acknowledge the contribution of the High-Performance Computing facility of the School of Computing Engineering and Mathematics, in the University of Brighton.

\section{REFERENCES}

[1] D. Mangini, M. Mameli, A. Georgoulas, L. Araneo, S. Filippeschi, and M. Marengo, “A pulsating heat pipe for space applications: Ground and microgravity experiments," Int. J. Therm. Sci., vol. 95, 2015.

[2] A. Georgoulas, P. Koukouvinis, M. Gavaises, and M. Marengo, "Numerical investigation of quasi-static bubble growth and detachment from submerged orifices in isothermal liquid pools: The effect of varying fluid properties and gravity levels," Int. J. Multiph. Flow, vol. 74, pp. 59-78, 2015.

[3] A. Georgoulas, M. Andredaki, and M. Marengo, "An Enhanced VOF Method Coupled with Heat Transfer and Phase Change to Characterise Bubble Detachment in Saturated Pool Boiling," Energies, vol. 10, no. 3, p. 272, Feb. 2017.

[4] J. . Brackbill, D. . Kothe, and C. Zemach, “A continuum method for modeling surface tension,” J. Comput. Phys., vol. 100, no. 2, pp. 335-354, Jun. 1992.

[5] D. A. Hoang, V. van Steijn, L. M. Portela, M. T. Kreutzer, and C. R. Kleijn, "Benchmark numerical simulations of segmented two-phase flows in microchannels using the Volume of Fluid method," Comput. Fluids, vol. 86, pp. 28-36, Nov. 2013.

[6] S. S. Deshpande, L. Anumolu, and M. F. Trujillo, "Evaluating the performance of the two-phase flow solver interFoam," Comput. Sci. Discov., vol. 5, no. 1, p. 14016, Nov. 2012.

[7] S. Hardt and F. Wondra, "Evaporation model for interfacial flows based on a continuum-field representation of the source terms," J. Comput. Phys., vol. 227, no. 11, pp. 5871-5895, 2008.

[8] E. Teodori, T. Valente, I. Malavasi, A. S. Moita, M. Marengo, and A. L. N. Moreira, "Effect of extreme wetting scenarios on pool boiling conditions," Appl. Therm. Eng., 2016.

[9] S. Arias, D. Legendre, and R. González-Cinca, "Numerical simulation of bubble generation in a T-junction," Comput. Fluids, vol. 56, pp. 49-60, 2012. 